\title{
Common Fixed Point Theorem for Weakly Compatible Mappings in Intuitionistic Fuzzy Metric Spaces
}

\author{
Anju Panwar, \\ Department of Mathematics, \\ Maharshi Dayanand University, \\ Rohtak, Haryana - 124001, India
}

\begin{abstract}
The present paper deals with a common fixed point theorem for weakly compatible mappings using condition of integral type in an intuitionistic fuzzy metric space. This result discovers the possibility of the idea of reciprocal continuity and weakly compatible maps to the problem of finding common fixed point of six mappings.
\end{abstract}

\section{Mathematics Subject Classification: 47H10, 54H25}

\section{Keywords}

Fuzzy set, Intuitionistic fuzzy set, Intuitionistic fuzzy metric space, weakly compatible, reciprocally continuous, Integral type

\section{INTRODUCTION}

In 1965, Zadeh [5 ]initiated the concept of fuzzy sets to describe the situation in which data are imprecise or vague or uncertain. After that in 1975, Kramosil and Michalek [6] gave the concept of fuzzy metric space, which opened an opportunity for further development of analysis in such spaces. Most of the early interest in fuzzy set theory pertained to representing uncertainty in human cognitive processes. Later on motivated by fuzzy sets, Atanassov [4] introduced and studied the concept of intuitionistic fuzzy sets. There has been much progress in the study of intuitionistic fuzzy sets by many authors. As a generalization of fuzzy metric space given by Kramosial and Michalek [6], Alaca, Turkoglu and Yildiz [3] proposed the idea of intuitionistic fuzzy metric spaces using t-norms and continuous t-conorms. Branciari [1] obtained a fixed point theorem for a single mapping satisfying an analogue of Banach's Contraction Principle for an integral type inequality. S.Muralisankar and G. Kalpana [7] proved a common fixed point theorem for pointwise R-weakly commuting mappings using contractive condition of integral type in an intuitionistic fuzzy metric space. The aim of this paper is to establish a common integral type fixed point theorem for weakly compatible mappings in an intuitionistic fuzzy metric space.

Definition 1.1. [ 5 ] Let $X$ be any set. A fuzzy set A in $X$ is a function with domain $\mathrm{X}$ and values in $[0,1]$.

Definition 1.2 [ 9 ]: A binary operation $*:[0,1] \times[0,1] \rightarrow[0$, $1]$ is a continuous t-norm if it satisfies the following conditions:

(1) * is associative and commutative,

(2) $*$ is continuous,

(3) $\mathrm{a} * 1=\mathrm{a}$ for all $\mathrm{a} \in[0,1]$,

(4) $\mathrm{a} * \mathrm{~b} \leq \mathrm{c} * \mathrm{~d}$ whenever $\mathrm{a} \leq \mathrm{c}$ and $\mathrm{b} \leq \mathrm{d}$, for each $a, b, c, d \in[0,1]$.

Example 1.3: Two typical examples of continuous t-norm are a $* \mathrm{~b}=\mathrm{ab}$ and $\mathrm{a} * \mathrm{~b}=\min (\mathrm{a}, \mathrm{b})$

Definition 1.4 [ 2 ] : A binary operation $\diamond:[0,1] \times[0,1] \rightarrow[0$, 1] is a continuous t-conorm if it satisfies the following conditions :

(1) $\diamond$ is associative and commutative,

(2) $\diamond$ is continuous,

(3) a $\diamond 0=$ a for all $\mathrm{a} \in[0,1]$,

(4) a $\diamond$ b $\leq$ c $\diamond$ d whenever $\mathrm{a} \leq \mathrm{c}$ and $\mathrm{b} \leq \mathrm{d}$, for each $\mathrm{a}, \mathrm{b}, \mathrm{c}, \mathrm{d} \in$ $[0,1]$.

Example 1.5 : Two typical examples of continuous t-conorm are $\mathrm{a} \diamond \mathrm{b}=\min (\mathrm{a}+\mathrm{b}, 1)$ and $\mathrm{a} \diamond \mathrm{b}=\max (\mathrm{a}, \mathrm{b})$.

Definition 1.6 [ 3 ] : A 5-tuple $(\mathrm{X}, \mathrm{M}, \mathrm{N}, *, \diamond)$ is called a intuitionistic fuzzy metric

space if $\mathrm{X}$ is an arbitrary (non-empty) set, * is a continuous tnorm, $\diamond$ a continuous $\mathrm{t}$-conorm and $\mathrm{M}, \mathrm{N}$ are fuzzy sets on $\mathrm{X}^{2} \times[0, \infty)$, satisfying the following conditions :

for each $\mathrm{x}, \mathrm{y}, \mathrm{z} \in \mathrm{X}$ and $\mathrm{t}, \mathrm{s}>0$,

(i)

$\mathrm{M}(\mathrm{x}, \mathrm{y}, \mathrm{t})+\mathrm{N}(\mathrm{x}, \mathrm{y}, \mathrm{t}) \leq 1$,

(ii) $\quad \mathrm{M}(\mathrm{x}, \mathrm{y}, 0)=0$

(iii) $\quad \mathrm{M}(\mathrm{x}, \mathrm{y}, \mathrm{t})=1$ if and only if $\mathrm{x}=\mathrm{y}$,

(iv) $\quad \mathrm{M}(\mathrm{x}, \mathrm{y}, \mathrm{t})=\mathrm{M}(\mathrm{y}, \mathrm{x}, \mathrm{t})$,

(v) $\quad \mathrm{M}(\mathrm{x}, \mathrm{y}, \mathrm{t}) * \mathrm{M}(\mathrm{y}, \mathrm{z}, \mathrm{s}) \leq \mathrm{M}(\mathrm{x}, \mathrm{z}, \mathrm{t}+\mathrm{s})$,

(vi) $\quad \mathrm{M}(\mathrm{x}, \mathrm{y},):.[0, \infty) \rightarrow[0,1]$ is left continuous,

(vii) $\lim _{t \rightarrow \infty} \mathrm{M}(\mathrm{x}, \mathrm{y}, \mathrm{t})=1$,

(viii) $\quad \mathrm{N}(\mathrm{x}, \mathrm{y}, 0)=1$,

(ix) $\quad \mathrm{N}(\mathrm{x}, \mathrm{y}, \mathrm{t})=0$ if and only if $\mathrm{x}=\mathrm{y}$,

(x) $\quad \mathrm{N}(\mathrm{x}, \mathrm{y}, \mathrm{t})=\mathrm{N}(\mathrm{y}, \mathrm{x}, \mathrm{t})$,

(xi) $\quad \mathrm{N}(\mathrm{x}, \mathrm{y}, \mathrm{t}) \diamond \mathrm{N}(\mathrm{y}, \mathrm{z}, \mathrm{s}) \geq \mathrm{N}(\mathrm{x}, \mathrm{z}, \mathrm{t}+\mathrm{s})$,

(xii) $\quad \mathrm{N}(\mathrm{x}, \mathrm{y},):.[0, \infty) \rightarrow[0,1]$ is right continuous,

(xiii) $\quad \lim _{t \rightarrow \infty} \mathrm{N}(\mathrm{x}, \mathrm{y}, \mathrm{t})=0$.

Then $(M, N)$ is called an intuitionistic fuzzy metric on $X$. The functions $M(x, y, t)$ and $N(x, y, t)$ denote the degree of nearness and the degree of non-nearness between $\mathrm{x}$ and $\mathrm{y}$ with respect to $\mathrm{t}$, respectively. 


\section{Example 1.7: (Induced intuitionistic fuzzy metric)}

Let $(\mathrm{X}, \mathrm{d})$ be a metric space. Denote $\mathrm{a} * \mathrm{~b}=\mathrm{ab}$ and $\mathrm{a} \diamond \mathrm{b}=\min (\mathrm{a}$ $+b, 1)$ for all $a, b \in[0,1]$ and let $M_{d}$ and $N_{d}$ be fuzzy sets on $\mathrm{X}^{2} \times(0, \infty)$ defined as follows:

$\mathrm{M}_{\mathrm{d}}(\mathrm{x}, \mathrm{y}, \mathrm{t})=\frac{h t^{n}}{h t^{n}+m(d(x, y))}$

$$
\mathrm{N}_{\mathrm{d}}(\mathrm{x}, \mathrm{y}, \mathrm{t})=\frac{d(x, y)}{k t^{n}+m(d(x, y))}
$$

for all $\mathrm{h}, \mathrm{k}, \mathrm{m}, \mathrm{n} \in \mathrm{R}^{+}$. Then $\left(\mathrm{X}, \mathrm{M}_{\mathrm{d}}, \mathrm{N}_{\mathrm{d}}, *, \diamond\right)$ is an intuitionistic fuzzy metric space.

Example1.8 : Let $\mathrm{X}=\{1\} \bigcup\left\{1-\frac{1}{n+1}: n \in N\right\}$ and *,

$\diamond$ be continuous t-norm and continuous t-conorm, respectively, defined by $\mathrm{a} * \mathrm{~b}=\mathrm{ab}$ and $\mathrm{a} \diamond \mathrm{b}=\min (\mathrm{a}+\mathrm{b}, 1)$ for all $\mathrm{a}, \mathrm{b} \in[0$, 1]. For each $x, y \in X$ and each $t>0$, define

$$
\mathrm{M}(\mathrm{x}, \mathrm{y}, \mathrm{t})=\left\{\begin{array}{lll}
1 & \text { if } & x=y \\
t x y & \text { if } & x \neq y \& t<1 \\
x y & \text { if } & x \neq y \& t \geq 1
\end{array}\right.
$$

and

$$
\mathrm{N}(\mathrm{x}, \mathrm{y}, \mathrm{t})=\left\{\begin{array}{lll}
0 & \text { if } & x=y \\
1-t x y & \text { if } & x \neq y \& t<1 \\
1-x y & \text { if } & x \neq y \& t \geq 1
\end{array} .\right.
$$

Then $(X, M, N, *, \diamond)$ is an intuitionistic fuzzy metric space

Definition 1.9[3] : A sequence $\left\{x_{n}\right\}$ in a intuitionistic fuzzy metric space $(\mathrm{X}, \mathrm{M}$,

$N, *, \diamond)$ converges to $x$ if and only if $M(x n, x, t) \rightarrow 1$ and $N(x n$, $\mathrm{x}, \mathrm{t}) \rightarrow 0$ as

$\mathrm{n} \rightarrow \infty$, for each $\mathrm{t}>0$.

It is called a Cauchy sequence if for each $0<\varepsilon<1$ and $t>0$, there exists $\mathrm{n}_{0} \in \mathrm{N}$ such that $\mathrm{M}\left(\mathrm{x}_{\mathrm{n}}, \mathrm{x}_{\mathrm{m}}, \mathrm{t}\right)>1-\varepsilon$ and $\mathrm{N}\left(\mathrm{x}_{\mathrm{n}}\right.$, $\left.\mathrm{x}_{\mathrm{m}}, \mathrm{t}\right)<\varepsilon$ for each $n, m \geq \mathrm{n}_{0}$.

The intuitionistic fuzzy metric space $(\mathrm{X}, \mathrm{M}, \mathrm{N}, *$, , .) is said to be complete if every Cauchy sequence is convergent.

Definition 1.10 [3]: Let $(X, M, N, * \diamond)$ be an intuitionistic fuzzy metric space and $\mathrm{A}, \mathrm{S}$ are mappings from $\mathrm{X}$ into itself. Then the mappings are said to be reciprocally continuous if

$$
\lim _{n \rightarrow \infty} \mathrm{ASx}_{\mathrm{n}}=\mathrm{Az} \quad \text { and } \quad \lim _{n \rightarrow \infty} \mathrm{SAx}_{\mathrm{n}}=\mathrm{Sz}
$$

whenever $\left\{x_{n}\right\}$ is a sequence in $X$ such that

$$
\lim _{n \rightarrow \infty} \mathrm{Ax}_{\mathrm{n}}=\lim _{n \rightarrow \infty} \mathrm{Sx}_{\mathrm{n}}=\mathrm{z} \text {, for some } \mathrm{z} \in \mathrm{X} .
$$

If $A$ and $S$ are both continuous then they are obviously reciprocally continuous. But the converse need not be true.
Definition 1.11 [3]: Self mappings A and B of an intuitionistic fuzzy metric space $(X, M, N, *, \diamond)$ is said to be weakly compatible if $\mathrm{ABx}=\mathrm{BAx}$ when $\mathrm{Ax}=\mathrm{Bx}$ for some $\mathrm{x} \in \mathrm{X}$.

It is easy to see that if self mappings $A$ and $B$ of an intuitionistic fuzzy metric space

$(\mathrm{X}, \mathrm{M}, \mathrm{N}, *, \diamond)$ is compatible then they are weakly compatible.

The following example shows that the converse of above statement does not hold.

Example 1.12: Let $(X, M, N, *, \diamond)$ be an intuitionistic fuzzy metric space, Where $X=[0,2]$ with t-norm and t-conorm defined by $\mathrm{a} * \mathrm{~b}=\min \{\mathrm{a}, \mathrm{b}\}$ and $\mathrm{a} \diamond \mathrm{b}=\max \{\mathrm{a}, \mathrm{b}\}$, for all $\mathrm{a}, \mathrm{b}$ $\in[0,1]$ and

$\mathrm{M}_{\mathrm{d}}(\mathrm{x}, \mathrm{y}, \mathrm{t})=\frac{t}{t+d(x, y)}, \mathrm{N}_{\mathrm{d}}(\mathrm{x}, \mathrm{y}, \mathrm{t})=\frac{d(x, y)}{t+d(x, y)}$

For all $\mathrm{t}>0$ and $\mathrm{M}_{\mathrm{d}}(\mathrm{x}, \mathrm{y}, \mathrm{t})=0$ and $\mathrm{N}_{\mathrm{d}}(\mathrm{x}, \mathrm{y}, \mathrm{t})=1$, for all $\mathrm{x}, \mathrm{y}$ $\in \mathrm{X}$.

Define self maps A and B on X as follows:

$\mathrm{Ax}=\left\{\begin{array}{lr}2 & \text { if } 0 \leq x \leq 1 \\ \frac{x}{2} & \text { if } 1<x \leq 2\end{array}\right.$

$\mathrm{Bx}=\left\{\begin{aligned} 2 & \text { if } x=1 \\ \frac{x+3}{5} & \text { otherwise }\end{aligned}\right.$

And $x_{n}=2-\frac{1}{(2 n)}$. Then we have

$\mathrm{A}(1)=\mathrm{B}(1)=2$ and $\mathrm{A}(2)=\mathrm{B}(2)=1$.

Also $\mathrm{AB}(1)=\mathrm{BA}(1)=2$. Thus $(\mathrm{A}, \mathrm{B})$ is weak compatible. Again

$\mathrm{Ax}_{\mathrm{n}}=1-\frac{1}{4 n}, \mathrm{Bx}_{\mathrm{n}}=1-\frac{1}{10 n}$

Thus $\mathrm{Ax}_{\mathrm{n}}=1, \mathrm{Bx}_{\mathrm{n}}=1$

Hence $\mathrm{z}=1$.

Further $\mathrm{ABx}_{\mathrm{n}}=2, \mathrm{BAx}_{\mathrm{n}}=\frac{4}{5}-\frac{1}{20 n}$

Now $\lim _{n \rightarrow \infty} \mathrm{M}\left(\mathrm{ABx}_{\mathrm{n}}, \mathrm{BAx}_{\mathrm{n}}, \mathrm{t}\right)=\lim _{\mathrm{n} \rightarrow \infty} \mathrm{M}\left(2, \frac{4}{5}-\frac{1}{20 n}, \mathrm{t}\right)$ $=\frac{t}{t+\frac{6}{5}} \neq 1$

$\lim _{n \rightarrow \infty} \mathrm{N}\left(\mathrm{ABx}_{\mathrm{n}}, \mathrm{BAx}_{\mathrm{n}}, \mathrm{t}\right)=\lim _{\mathrm{n} \rightarrow \infty} \mathrm{N}\left(2, \frac{4}{5}-\frac{1}{20 n}, \mathrm{t}\right)$ $=\frac{\frac{6}{5}}{t+\frac{6}{5}} \neq 0, \mathrm{t}>0$ 
Hence (A, B) is not compatible.

Lemma 1.13. [8] Let $(X, M, N, *, \diamond)$ be an intuitionistic fuzzy metric space.

If there exists a constant $\mathrm{k} \in(0,1)$ such that

$$
\begin{aligned}
& M\left(y_{n+2}, y_{n+1}, k t\right) \geq M\left(y_{n+1}, y_{n}, t\right), \\
& N\left(y_{n+2}, y_{n+1}, k t\right) \leq N\left(y_{n+1}, y_{n}, t\right),
\end{aligned}
$$

$\forall \mathrm{t}>0$ and $\mathrm{n}=1,2, .$. then $\left\{\mathrm{y}_{\mathrm{n}}\right\}$ is a Cauchy sequence in $\mathrm{X}$.

Lemma 1.14 [ 8 ] Let $(\mathrm{X}, \mathrm{M}, \mathrm{N}, *, \diamond)$ be an intuitionistic fuzzy metric space.

If there exists a constant $\mathrm{k} \in(0,1)$ such that

$$
\begin{aligned}
& M(x, y, k t) \geq M(x, y, t), \\
& N(x, y, k t) \leq N(x, y, t),
\end{aligned}
$$

for $\mathrm{x}, \mathrm{y} \in \mathrm{X}$. Then $\mathrm{x}=\mathrm{y}$.

\section{MAIN RESULT}

Theorem 2.1 Let $\mathrm{P}, \mathrm{Q}, \mathrm{A}, \mathrm{B}, \mathrm{S}$ and $\mathrm{T}$ be self mappings of a complete intuitionistic fuzzy metric space $(\mathrm{X}, \mathrm{M}, \mathrm{N}, *, \diamond)$ with continuous t-norm $*$ and continuous t-corm $\diamond$ defined by $\mathrm{t}$ $* t \geq t$ and (1-t) $\diamond(1-t) \leq(1-t)$ for all $\mathrm{t} \in[0,1]$ such that

(i) $\mathrm{P}(\mathrm{X}) \subseteq \mathrm{ST}(\mathrm{X}), \mathrm{Q}(\mathrm{X}) \subseteq \mathrm{AB}(\mathrm{X})$

(ii) there exists a constant $\mathrm{k} \in(0,1)$ such that

$$
\begin{array}{r}
\int_{0}^{M(P x, Q y, k t)} \phi(t) d t \geq\left(\int_{0}^{m(x, y, t)} \phi(t) d t\right), \\
\int_{0}^{N(P x, Q y, k t)} \phi(t) d t \leq\left(\int_{0}^{n(x, y, t)} \phi(t) d t\right),
\end{array}
$$

where $\varphi: \mathrm{R}^{+} \rightarrow \mathrm{R}^{+}$is a Lebesgue- integrable mappings which is summable, nonnegative, and

such that

$$
\int_{0}^{\epsilon} \varphi(t) d t>0
$$

for each $\in>0$,

where

$$
\begin{aligned}
\mathrm{m}(\mathrm{x}, \mathrm{y}, \mathrm{t})=\min \{\mathrm{M}(\mathrm{STy}, \mathrm{Qy}, \mathrm{t}), \mathrm{M}(\mathrm{ABx}, \mathrm{Px}, \mathrm{t}), \\
\mathrm{M}(\mathrm{ABx}, \mathrm{Qy}, \alpha \mathrm{t}), \mathrm{M}(\mathrm{STy}, \mathrm{Px},(2-\alpha) \mathrm{t}), \\
\mathrm{M}(\mathrm{STy}, \mathrm{ABx}, \mathrm{t})\} \\
\mathrm{n}(\mathrm{x}, \mathrm{y}, \mathrm{t})=\max \{\mathrm{N}(\mathrm{STy}, \mathrm{Qy}, \mathrm{t}), \\
\mathrm{N}(\mathrm{ABx}, \mathrm{Px}, \mathrm{t}), \mathrm{N}(\mathrm{ABx}, \mathrm{Qy}, \alpha \mathrm{t}), \\
\mathrm{N}(\mathrm{STy}, \mathrm{Px},(2-\alpha) \mathrm{t}, \mathrm{N}(\mathrm{STy}, \mathrm{Px}, \mathrm{t}))\}
\end{aligned}
$$

for all $\mathrm{x}, \mathrm{y} \in \mathrm{X}, \alpha \in(0,2)$ and $\mathrm{t}>0$. (iii)(a) $(\mathrm{P}, \mathrm{AB})$ is reciprocally continuous and $(\mathrm{Q}, \mathrm{ST})$ is weakly compatible or

(iii)(b) $(\mathrm{Q}, \mathrm{ST})$ is reciprocally continuous and $(\mathrm{P}, \mathrm{AB})$ is weakly compatible.

(iv) $\mathrm{AB}=\mathrm{BA}, \mathrm{ST}=\mathrm{TS}, \mathrm{PB}=\mathrm{BP}, \mathrm{SQ}=\mathrm{QS}, \mathrm{QT}=\mathrm{TQ}$.

(v) A, B, S and T are continuous. fixed point.

Then $\mathrm{P}, \mathrm{Q}, \mathrm{A}, \mathrm{B}, \mathrm{S}$ and $\mathrm{T}$ have a unique common

Proof. Let $\mathrm{x}_{0}$ be any point in $\mathrm{X}$. We construct a sequence $\left\{\mathrm{y}_{\mathrm{n}}\right\}$ in $\mathrm{X}$ such that for $\mathrm{n}=0,1,2 \ldots$.

$$
\begin{gathered}
y_{2 n}=\operatorname{Px}_{2 n}=\operatorname{STx}_{2 n+1} \\
\mathrm{y}_{2 \mathrm{n}+1}=\mathrm{Qx}_{2 \mathrm{n}+1}=\mathrm{ABx}_{2 \mathrm{n}+2}
\end{gathered}
$$

We show that $\left\{y_{n}\right\}$ is a Cauchy sequence. By (2.1) and (2.2), for all $\mathrm{t}>0$ and $\alpha=1-\beta$ with $\beta \in(0,1)$, we have

$$
\begin{gathered}
\int_{0}^{M\left(y_{2 n+1}, y_{2 n+2}, k t\right)} \phi(t) d t=\int_{0}^{M\left(Q x_{2 n+1}, P x_{2 n+2}, k t\right)} \phi(t) d t, \\
=\int_{0}^{M\left(P x_{2 n+2}, Q x_{2 n+1}, k t\right)} \varphi(t) d t, \\
\geq \int_{0}^{m\left(x_{2 n+2}, x_{2 n+1}, t\right)} \phi(t) d t,
\end{gathered}
$$

$$
\begin{aligned}
\int_{0}^{N\left(y_{2 n+1}, y_{2 n+2}, k t\right)} \phi(t) d t & =\int_{0}^{N\left(Q x_{2 n+1}, P x_{2 n+2}, k t\right)} \phi(t) d t \\
& =\int_{0}^{N\left(P x_{2 n+2}, Q x_{2 n+1}, k t\right)} \varphi(t) d t,
\end{aligned}
$$

$$
\begin{aligned}
& \leq \quad \int_{0}^{n\left(x_{2 n+2}, x_{2 n+1}, t\right)} \varphi(t) d t . \\
& \mathrm{m}\left(\mathrm{x}_{2 \mathrm{n}+2}, \mathrm{x}_{2 \mathrm{n}+1}, \mathrm{t}\right)=\min \left\{\mathrm{M}\left(\mathrm{STx}_{2 \mathrm{n}+1}, \mathrm{Qx}_{2 \mathrm{n}+1}, \mathrm{t}\right),\right. \\
& \mathrm{M}\left(\mathrm{Px}_{2 \mathrm{n}+2}, \mathrm{ABx}_{2 \mathrm{n}+2}, \mathrm{t}\right), \mathrm{M}\left(\mathrm{ABx}_{2 \mathrm{n}+2}, \mathrm{Qx}_{2 \mathrm{n}+1}, \alpha \mathrm{t}\right), \\
& \mathrm{M}\left(\mathrm{STx}_{2 \mathrm{n}+1}, \mathrm{Px}_{2 \mathrm{n}+2},(2-\alpha) \mathrm{t}\right), \\
& \left.\mathrm{M}\left(\mathrm{STx}_{2 \mathrm{n}+1}, \mathrm{ABx}_{2 \mathrm{n}+2}, \mathrm{t}\right)\right\} \\
& =\min \left\{\mathrm{M}\left(\mathrm{y}_{2 \mathrm{n}}, \mathrm{y}_{2 \mathrm{n}+1}, \mathrm{t}\right), \mathrm{M}\left(\mathrm{y}_{2 \mathrm{n}+1}, \mathrm{y}_{2 \mathrm{n}+2}, \mathrm{t}\right)\right. \text {, } \\
& \mathrm{M}\left(\mathrm{y}_{2 \mathrm{n}+1}, \mathrm{y}_{2 \mathrm{n}+1}, \alpha \mathrm{t}\right), \mathrm{M}\left(\mathrm{y}_{2 \mathrm{n}}, \mathrm{y}_{2 \mathrm{n}+2},(1+\beta) \mathrm{t}\right), \\
& \left.\mathrm{M}\left(\mathrm{y}_{2 \mathrm{n}}, \mathrm{y}_{2 \mathrm{n}+1}, \mathrm{t}\right)\right\} \\
& \geq \min \left\{\mathrm{M}\left(\mathrm{y}_{2 \mathrm{n}}, \mathrm{y}_{2 \mathrm{n}+1}, \mathrm{t}\right), \mathrm{M}\left(\mathrm{y}_{2 \mathrm{n}+1}, \mathrm{y}_{2 \mathrm{n}+2}, \mathrm{t}\right),\right. \\
& \mathrm{M}\left(\mathrm{y}_{2 \mathrm{n}}, \mathrm{y}_{2 \mathrm{n}+1}, \mathrm{t}\right), \mathrm{M}\left(\mathrm{y}_{2 \mathrm{n}+1}, \mathrm{y}_{2 \mathrm{n}+2}, \beta \mathrm{t}\right), \\
& \left.\mathrm{M}\left(\mathrm{y}_{2 \mathrm{n}}, \mathrm{y}_{2 \mathrm{n}+1}, \mathrm{t}\right)\right\}
\end{aligned}
$$




$$
\geq \min \left\{\mathrm{M}\left(\mathrm{y}_{2 \mathrm{n}}, \mathrm{y}_{2 \mathrm{n}+1}, \mathrm{t}\right), \mathrm{M}\left(\mathrm{y}_{2 \mathrm{n}+1}, \mathrm{y}_{2 \mathrm{n}+2}, \mathrm{t}\right),\right.
$$

$$
\left.\mathrm{M}\left(\mathrm{y}_{2 \mathrm{n}+1}, \mathrm{y}_{2 \mathrm{n}+2}, \beta \mathrm{t}\right)\right\}
$$

$\mathrm{n}\left(\mathrm{x}_{2 \mathrm{n}+2}, \mathrm{x}_{2 \mathrm{n}+1}, \mathrm{t}\right)=\max \left\{\mathrm{N}\left(\mathrm{STx}_{2 \mathrm{n}+1}, \mathrm{Qx} \mathrm{x}_{2 \mathrm{n}+1}, \mathrm{t}\right), \mathrm{N}\left(\mathrm{Px}_{2 \mathrm{n}+2}, \mathrm{ABx}_{2 \mathrm{n}+2}, \mathrm{t}\right)\right.$,

$\mathrm{N}\left(\mathrm{ABx}_{2 \mathrm{n}+2}, \mathrm{Qx}_{2 \mathrm{n}+1}, \alpha \mathrm{t}\right)$,

$\mathrm{N}\left(\operatorname{STx}_{2 \mathrm{n}+1}, \mathrm{Px}_{2 \mathrm{n}+2},(2-\alpha) \mathrm{t}\right)$,

$\left.\mathrm{N}\left(\mathrm{STx}_{2 \mathrm{n}+1}, \mathrm{ABx}_{2 \mathrm{n}+2}, \mathrm{t}\right)\right\}$

$=\max \left\{\mathrm{N}\left(\mathrm{y}_{2 \mathrm{n}}, \mathrm{y}_{2 \mathrm{n}+1}, \mathrm{t}\right), \mathrm{N}\left(\mathrm{y}_{2 \mathrm{n}+1}, \mathrm{y}_{2 \mathrm{n}+2}, \mathrm{t}\right)\right.$,

$\mathrm{N}\left(\mathrm{y}_{2 \mathrm{n}+1}, \mathrm{y}_{2 \mathrm{n}+1}, \alpha \mathrm{t}\right), \mathrm{N}\left(\mathrm{y}_{2 \mathrm{n}}, \mathrm{y}_{2 \mathrm{n}+2},(1+\beta) \mathrm{t}\right)$,

$\left.\mathrm{N}\left(\mathrm{y}_{2 \mathrm{n}}, \mathrm{y}_{2 \mathrm{n}+1}, \mathrm{t}\right)\right\}$

$\leq \max \left\{\mathrm{N}\left(\mathrm{y}_{2 \mathrm{n}}, \mathrm{y}_{2 \mathrm{n}+1}, \mathrm{t}\right), \mathrm{N}\left(\mathrm{y}_{2 \mathrm{n}+1}, \mathrm{y}_{2 \mathrm{n}+2}, \mathrm{t}\right)\right.$,

$1, \mathrm{~N}\left(\mathrm{y}_{2 \mathrm{n}}, \mathrm{y}_{2 \mathrm{n}+1}, \mathrm{t}\right), \mathrm{N}\left(\mathrm{y}_{2 \mathrm{n}+1}, \mathrm{y}_{2 \mathrm{n}+2}, \beta \mathrm{t}\right)$,

$\left.\mathrm{N}\left(\mathrm{y}_{2 \mathrm{n}}, \mathrm{y}_{2 \mathrm{n}+1}, \mathrm{t}\right)\right\}$

$\leq \max \left\{\mathrm{N}\left(\mathrm{y}_{2 \mathrm{n}}, \mathrm{y}_{2 \mathrm{n}+1}, \mathrm{t}\right), \mathrm{N}\left(\mathrm{y}_{2 \mathrm{n}+1}, \mathrm{y}_{2 \mathrm{n}+2}, \beta \mathrm{t}\right)\right.$,

$\left.\mathrm{N}\left(\mathrm{y}_{2 \mathrm{n}+1}, \mathrm{y}_{2 \mathrm{n}+2}, \mathrm{t}\right)\right\}$

since t-norm $*$, t-conorm $\diamond, \mathrm{M}(\mathrm{x}, \mathrm{y},$.$) and \mathrm{N}(\mathrm{x}, \mathrm{y},$.$) is$ continuous. Letting $\beta \rightarrow 1$, we have

$$
\begin{aligned}
& \mathrm{m}\left(\mathrm{x}_{2 \mathrm{n}+2}, \mathrm{x}_{2 \mathrm{n}+1}, \mathrm{t}\right) \geq \min \left\{\mathrm{M}\left(\mathrm{y}_{2 \mathrm{n}}, \mathrm{y}_{2 \mathrm{n}+1}, \mathrm{t}\right),\right. \\
&\left.\mathrm{M}\left(\mathrm{y}_{2 \mathrm{n}+1}, \mathrm{y}_{2 \mathrm{n}+2}, \mathrm{t}\right)\right\} \\
& \mathrm{n}\left(\mathrm{x}_{2 \mathrm{n}+2}, \mathrm{x}_{2 \mathrm{n}+1}, \mathrm{t}\right) \leq \max \left\{\mathrm{N}\left(\mathrm{y}_{2 \mathrm{n}}, \mathrm{y}_{2 \mathrm{n}+1}, \mathrm{t}\right),\right. \\
&\left.\mathrm{N}\left(\mathrm{y}_{2 \mathrm{n}+1}, \mathrm{y}_{2 \mathrm{n}+2}, \mathrm{t}\right)\right\}
\end{aligned}
$$

Therefore,

$$
\int_{0}^{M\left(y_{2 n+1}, y_{2 n+2}, k t\right)} \varphi(t) d t \geq \int_{0}^{\min M\left\{\left(y_{2 n}, y_{2 n+1}, t\right), M\left(y_{2 n+1}, y_{2 n+2}, t\right)\right\}} \varphi(t) d t,
$$

$$
\int_{0}^{N\left(y_{2 n+1}, y_{2 n+2}, k t\right)} \varphi(t) d t \leq \int_{0}^{\max N\left\{\left(y_{2 n}, y_{2 n+1}, t\right), N\left(y_{2 n+1}, y_{2 n+2}, t\right)\right\}} \varphi(t) d t
$$

Similarly, we can obtain

$$
\begin{gathered}
M\left(y_{2 n+2}, y_{2 n+3}, k t\right) d t \\
\int_{0}^{\min M\left\{\left(y_{2 n+1}, y_{2 n+2}, t\right), M\left(y_{2 n+2}, y_{2 n+3}, t\right)\right\}}, \\
\int_{0}^{N\left(y_{2 n+2}, y_{2 n+3}, k t\right)} \varphi(t) d t
\end{gathered}
$$

In general,

$$
\int_{0}^{M\left(y_{n+1}, y_{n+2}, k t\right)} \varphi(t) d t \geq \int_{0}^{\min M\left\{\left(y_{n}, y_{n+1}, t\right), M\left(y_{n+1}, y_{n+2}, t\right)\right\}} \varphi(t) d t,
$$

$$
\int_{0}^{N\left(y_{n+1}, y_{n+2}, k t\right)} \varphi(t) d t \leq \int_{0}^{\max N\left\{\left(y_{n}, y_{n+1}, t\right), N\left(y_{n+1}, y_{n+2}, t\right)\right\}} \varphi(t) d t .
$$

and, for every positive integer $\mathrm{p}$,

$$
\int_{0}^{M\left(y_{n+1}, y_{n+2}, k t\right)} \varphi(t) d t \geq \quad \int_{0}^{\min M\left\{\left(y_{n}, y_{n+1}, t\right), M\left(y_{n+1}, y_{n+2}, t / k^{p}\right)\right\}}
$$

$\int_{0}^{N\left(y_{n+1}, y_{n+2}, k t\right)} \varphi(t) d t \leq \int_{0}^{\max N\left\{\left(y_{n}, y_{n+1}, t\right), N\left(y_{n+1}, y_{n+2}, t / k^{p}\right)\right\}} \varphi(t) d t$.

since $\mathrm{M}\left(\mathrm{y}_{\mathrm{n}+1}, \mathrm{y}_{\mathrm{n}+2}, \mathrm{t} / \mathrm{k}^{\mathrm{p}}\right) \rightarrow 1$ as $\mathrm{p} \rightarrow \infty, \mathrm{N}\left(\mathrm{y}_{\mathrm{n}+1}, \mathrm{y}_{\mathrm{n}+2}, \mathrm{t} / \mathrm{k}^{\mathrm{p}}\right) \rightarrow 0$ as $\mathrm{p} \rightarrow \infty$,

$$
\begin{array}{r}
M\left(y_{n+1}, y_{n+2}, k t\right) \\
\int_{0}^{M\left(y_{n}, y_{n+1}, t\right)} \varphi(t) d t \geq \int_{0}^{n} \varphi(t) d t . \\
\int_{0}^{N\left(y_{n+1}, y_{n+2}, k t\right)} \varphi(t) d t \leq \int_{0}^{N\left(y_{n}, y_{n+1}, t\right)} \varphi(t) d t .
\end{array}
$$

By Lemma(1.14), $\left\{y_{n}\right\}$ is Cauchy sequence in $X$. Since $X$ is a complete, there is a point $\mathrm{z}$ in $\mathrm{X}$ such that $\mathrm{y}_{\mathrm{n}} \rightarrow \mathrm{z} \in \mathrm{X}$.Hence from (2.3), we have

$$
\begin{aligned}
& y_{2 n}=S T x_{2 n+1}=P x_{2 n} \rightarrow z \\
& y_{2 n+1}=Q x_{2 n+1}=Q x_{2 n+2} \rightarrow z
\end{aligned}
$$

Since $\mathrm{P}$ and $\mathrm{AB}$ are compatible and reciprocally continuous mappings, then $\mathrm{PABx}_{2 \mathrm{n}} \rightarrow \mathrm{Pz}$ and $\mathrm{ABPx}_{2 \mathrm{n}} \rightarrow \mathrm{ABz}$ as $\mathrm{n}$ $\rightarrow \infty$. The compatibility of the pair $(\mathrm{P}, \mathrm{AB})$ yields

$$
\lim _{n \rightarrow \infty} \mathrm{M}\left(\mathrm{PABx}_{2 \mathrm{n}}, \mathrm{ABPx}_{2 \mathrm{n}}, \mathrm{t}\right)=1
$$

That is,

$\mathrm{M}(\mathrm{Pz}, \mathrm{ABz}, \mathrm{t})=1$. Hence $\mathrm{Pz}=\mathrm{ABz}$.

The compatibility of the pair $(\mathrm{P}, \mathrm{AB})$ yields

$$
\lim _{n \rightarrow \infty} \mathrm{N}\left(\mathrm{PABx}_{2 \mathrm{n}}, \mathrm{ABPx}_{2 \mathrm{n}}, \mathrm{t}\right)=0
$$

That is,

$$
\mathrm{N}(\mathrm{Pz}, \mathrm{ABz}, \mathrm{t})=0 \text {. Hence } \mathrm{Pz}=\mathrm{ABz} .
$$

Since PX $\subset$ STX, there exist $\mathrm{w} \in \mathrm{X}$

$$
\text { such that } \mathrm{Pz}=\mathrm{STw}
$$

Using(ii), we get 


$$
\begin{array}{ccc}
\int_{0}^{M(P z, Q w, k t)} \varphi(t) d t & \geq \int_{0}^{m(z, w, t)} \varphi(t) d t \\
\int_{0}^{N(P z, Q w, k t)} \varphi(t) d t & \leq & \int_{0}^{n(z, w, t)} \varphi(t) d t .
\end{array}
$$

Take $\alpha=1$,

$\mathrm{m}(\mathrm{z}, \mathrm{w}, \mathrm{t})=\min \{\mathrm{M}(\mathrm{STw}, \mathrm{Qw}, \mathrm{t}), \mathrm{M}(\mathrm{ABz}, \mathrm{Pz}, \mathrm{t})$

$$
\begin{aligned}
& \mathrm{M}(\mathrm{ABz}, \mathrm{Qw}, \mathrm{t}), \mathrm{M}(\mathrm{STw}, \mathrm{Pz}, \mathrm{t}), \\
& \mathrm{M}(\mathrm{STw}, \mathrm{ABz}, \mathrm{t})\} \\
= & \min \{\mathrm{M}(\mathrm{Pz}, \mathrm{Qw}, \mathrm{t}), 1, \mathrm{M}(\mathrm{Pz}, \mathrm{Qw}, \mathrm{t}), 1,1\} \\
= & \min \{\mathrm{M}(\mathrm{Pz}, \mathrm{Qw}, \mathrm{t}), 1\},
\end{aligned}
$$

$\mathrm{n}(\mathrm{z}, \mathrm{w}, \mathrm{t})=\max \{\mathrm{N}(\mathrm{STw}, \mathrm{Qw}, \mathrm{t}), \mathrm{N}(\mathrm{ABz}, \mathrm{Pz}, \mathrm{t})$

$\mathrm{N}(\mathrm{ABz}, \mathrm{Qw}, \mathrm{t}), \mathrm{N}(\mathrm{STw}, \mathrm{Pz}, \mathrm{t})$,

$\mathrm{N}(\mathrm{STw}, \mathrm{ABz}, \mathrm{t})\}$

$=\max \{\mathrm{N}(\mathrm{Pz}, \mathrm{Qw}, \mathrm{t}), 0, \mathrm{~N}(\mathrm{Pz}, \mathrm{Qw}, \mathrm{t}), 0,0\}$

$=\max \{\mathrm{N}(\mathrm{Pz}, \mathrm{Qw}, \mathrm{t}), 0\}$.

$$
\int_{0}^{M(P z, Q w, k t)} \varphi(t) d t \geq \int_{0}^{M(P z, Q w, t)} \varphi(t) d t,
$$$$
\int_{0}^{N(P z, Q w, k t)} \varphi(t) d t \leq \int_{0}^{N(P z, Q w, t)} \varphi(t) d t .
$$

By using Lemma,1.14, we get $\mathrm{Pz}=\mathrm{Qw}$

(2.7).

Thus,

$$
\mathrm{ABz}=\mathrm{Pz}=\mathrm{Qw}=\mathrm{STw} .
$$

We now claim that $\mathrm{Pz}=\mathrm{z}$, for this we take $\mathrm{x}=\mathrm{z}, \quad \mathrm{y}=\mathrm{x}_{2 \mathrm{n}-1}$ and using (ii), we get

$$
\begin{aligned}
& \int_{0}^{M\left(P z, x_{2 n-1}, k t\right)} \varphi(t) d t \geq \int_{0}^{m\left(z, x_{2 n-1}, t\right)} \varphi(t) d t, \\
& \int_{0}^{N\left(P z, x_{2 n-1}, k t\right)} \varphi(t) d t \leq \int_{0}^{n\left(z, x_{2 n-1}, t\right)} \varphi(t) d t . \\
& \int_{0}^{N\left(P z, x_{2 n-1}, k t\right)} \varphi(t) d t \leq \int_{0}^{n\left(z, x_{2 n-1}, t\right)} \varphi(t) d t . \quad \text { Take } \alpha=1 \\
& \mathrm{~m}\left(\mathrm{z}, \mathrm{x}_{2 \mathrm{n}-1}, \mathrm{t}\right)=\min \left\{\mathrm{M}\left(\mathrm{STx}_{2 \mathrm{n}-1}, \mathrm{ABx} \mathrm{x}_{2 \mathrm{n}-1}, \mathrm{t}\right),\right. \\
& \mathrm{M}(\mathrm{ABz}, \mathrm{Pz}, \mathrm{t}), \mathrm{M}\left(\mathrm{ABz}, \mathrm{Qx}_{2 \mathrm{n}-1}, \mathrm{t}\right) \text {, } \\
& \left.\mathrm{M}\left(\mathrm{STx}_{2 \mathrm{n}-1}, \mathrm{Pz}, \mathrm{t}\right) \mathrm{M}\left(\mathrm{STx}_{2 \mathrm{n}-1}, \mathrm{ABz}, \mathrm{t}\right)\right\} \\
& =\min \{\mathrm{M}(\mathrm{z}, \mathrm{z}, \mathrm{t}), \mathrm{M}(\mathrm{Pz}, \mathrm{Pz}, \mathrm{t}), \\
& \mathrm{M}(\mathrm{ABz}, \mathrm{z}, \mathrm{t}), \mathrm{M}(\mathrm{z}, \mathrm{Pz}, \mathrm{t}), \mathrm{M}(\mathrm{z}, \mathrm{ABz}, \mathrm{t})\}
\end{aligned}
$$

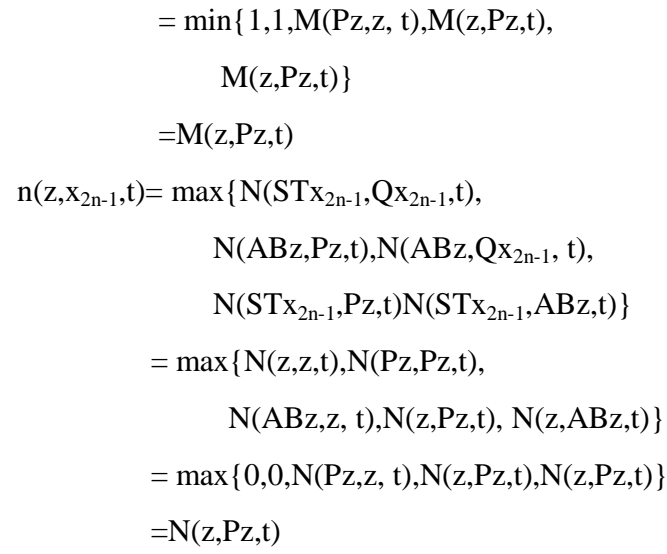

By using Lemma 1.14,we get $\mathrm{Pz}=\mathrm{z}$

Thus,

$$
\mathrm{z}=\mathrm{Pz}=\mathrm{ABz}=\mathrm{Qw}=\mathrm{ST}
$$

Since the pair (Q,ST) is weakly compatible,we have

$$
\begin{aligned}
\mathrm{QSTw} & =\mathrm{STQw}, \text { we get } \\
\mathrm{Qz} & =\mathrm{STz} .
\end{aligned}
$$

Now we claim that $\mathrm{Qz}=\mathrm{Pz}$.

Using (ii),we get

$$
\begin{aligned}
\int_{0}^{M(P z, Q z, k t)} \varphi(t) d t & \geq \int_{0}^{m(z, z, t)} \varphi(t) d t, \\
\int_{0}^{N(P z, Q z, k t)} \phi(t) d t & \leq \int_{0}^{n(z, z, t)} \phi(t) d t .
\end{aligned}
$$

$\int_{0}^{N(P z, Q z, k t)} \phi(t) d t \leq \int_{0}^{n(z, z, t)} \phi(t) d t$

Take $\alpha=1$

$\mathrm{m}(\mathrm{z}, \mathrm{z}, \mathrm{t})=\min \{\mathrm{M}(\mathrm{STz}, \mathrm{Qz}, \mathrm{t}), \mathrm{M}(\mathrm{ABz}, \mathrm{Pz}, \mathrm{t})$,

$\mathrm{M}(\mathrm{ABz}, \mathrm{Qz}, \mathrm{t}), \mathrm{M}(\mathrm{STz}, \mathrm{Pz}, \mathrm{t})$

$\mathrm{M}(\mathrm{STz}, \mathrm{ABz}, \mathrm{t})\}$

$=\min \{\mathrm{M}(\mathrm{Qz}, \mathrm{Qz}, \mathrm{t}), \mathrm{M}(\mathrm{Pz}, \mathrm{Pz}, \mathrm{t})$

$\mathrm{M}(\mathrm{Pz}, \mathrm{Qz}, \mathrm{t}), \mathrm{M}(\mathrm{Qz}, \mathrm{Pz}, \mathrm{t}), \mathrm{M}(\mathrm{Qz}, \mathrm{Pz}, \mathrm{t})\}$

$=\min \{1,1, \mathrm{M}(\mathrm{Pz}, \mathrm{Qz}, \mathrm{t}), \mathrm{M}(\mathrm{Qz}, \mathrm{Pz}, \mathrm{t})$,

$\mathrm{M}(\mathrm{Qz}, \mathrm{Pz}, \mathrm{t})\}$

$=\mathrm{M}(\mathrm{Pz}, \mathrm{Qz}, \mathrm{t})$

$\mathrm{n}(\mathrm{z}, \mathrm{z}, \mathrm{t})=\max \{\mathrm{N}(\mathrm{STz}, \mathrm{Qz}, \mathrm{t}), \mathrm{N}(\mathrm{ABz}, \mathrm{Pz}, \mathrm{t})$,

$$
\begin{gathered}
\mathrm{N}(\mathrm{ABz}, \mathrm{Qz}, \mathrm{t}), \mathrm{N}(\mathrm{STz}, \mathrm{Pz}, \mathrm{t}) \\
\mathrm{N}(\mathrm{STz}, \mathrm{ABz}, \mathrm{t})\} \\
=\max \{\mathrm{N}(\mathrm{Qz}, \mathrm{Qz}, \mathrm{t}), \mathrm{N}(\mathrm{Pz}, \mathrm{Pz}, \mathrm{t}), \\
\mathrm{N}(\mathrm{Pz}, \mathrm{Qz}, \mathrm{t}), \mathrm{N}(\mathrm{Qz}, \mathrm{Pz}, \mathrm{t}), \\
\mathrm{N}(\mathrm{Qz}, \mathrm{Pz}, \mathrm{t})\}
\end{gathered}
$$




$$
\begin{aligned}
& =\max \{0,0, \mathrm{~N}(\mathrm{Pz}, \mathrm{Qz}, \mathrm{t}), \\
& \quad \mathrm{N}(\mathrm{Qz}, \mathrm{Pz}, \mathrm{t}), \mathrm{N}(\mathrm{Qz}, \mathrm{Pz}, \mathrm{t})\} \\
& =\mathrm{N}(\mathrm{Pz}, \mathrm{Qz}, \mathrm{t})
\end{aligned}
$$

By using Lemma, we get $\mathrm{Pz}=\mathrm{Qz}$.

We now claim that $\mathrm{Tz}=\mathrm{z}$, for this we take $\mathrm{x}=\mathrm{x}_{2 \mathrm{n}}, \mathrm{y}=\mathrm{Tz}$ and using (ii), we get

$$
\begin{gathered}
\int_{0}^{M\left(P x_{2 n}, Q T z, k t\right)} \varphi(t) d t \geq \int_{0}^{m\left(x_{2 n}, T z, t\right)} \varphi(t) d t, \\
\int_{0}^{N\left(P x_{2 n}, Q T z, k t\right)} \varphi(t) d t \geq \int_{0}^{n\left(x_{2 n}, T z, t\right)} \varphi(t) d t \\
\int_{0}^{N\left(P x_{2 n}, Q T z, k t\right)} \varphi(t) d t \leq \int_{0}^{n\left(x_{2 n}, T z, t\right)} \varphi(t) d t .
\end{gathered}
$$

$\mathrm{m}\left(\mathrm{x}_{2 \mathrm{n}}, \mathrm{Tz}, \mathrm{t}\right)=\min \left\{\mathrm{M}(\mathrm{STT} z, \mathrm{QTz}, \mathrm{t}), \mathrm{M}\left(\mathrm{ABx}_{2 \mathrm{n}}, \mathrm{Px}_{2 \mathrm{n}}, \mathrm{t}\right)\right.$,

$\mathrm{M}\left(\mathrm{ABx}_{2 \mathrm{n}}, \mathrm{QTz}, \mathrm{t}\right), \mathrm{M}\left(\mathrm{STTz}_{\mathrm{N}} \mathrm{Px}_{2 \mathrm{n}}, \mathrm{t}\right)$,

$\left.\mathrm{M}\left(\mathrm{STTz}, \mathrm{ABx}_{2 \mathrm{n}}, \mathrm{t}\right)\right\}$

As $\mathrm{QT}=\mathrm{TQ}$ and $\mathrm{ST}=\mathrm{TS}$ we have $\mathrm{QTz}=\mathrm{TQz}=\mathrm{Tz}$ and

$\mathrm{ST}(\mathrm{Tz})=\mathrm{T}(\mathrm{ST}) \mathrm{z}=\mathrm{Tz}$.

Letting $n \rightarrow \infty$ we get,

$\mathrm{m}(\mathrm{z}, \mathrm{Tz}, \mathrm{t})=\min \{\mathrm{M}(\mathrm{Tz}, \mathrm{Tz}, \mathrm{t}), \mathrm{M}(\mathrm{ABz}, \mathrm{Pz}, \mathrm{t})$,

$$
\begin{aligned}
& \mathrm{M}(\mathrm{ABz}, \mathrm{Tz}, \mathrm{t}), \mathrm{M}(\mathrm{Tz}, \mathrm{Pz}, \mathrm{t}) \text {, } \\
& \mathrm{M}(\mathrm{Tz}, \mathrm{ABz}, \mathrm{t})\} \\
& =\min \{1, \mathrm{M}(\mathrm{z}, \mathrm{z}, \mathrm{t}), \mathrm{M}(\mathrm{z}, \mathrm{Tz}, \mathrm{t}), \mathrm{M}(\mathrm{Tz}, \mathrm{z}, \mathrm{t}) \text {, } \\
& \mathrm{M}(\mathrm{Tz}, \mathrm{z}, \mathrm{t})\} \\
& =\min \{1,0, \mathrm{M}(\mathrm{z}, \mathrm{Tz}, \mathrm{t}), \mathrm{M}(\mathrm{Tz}, \mathrm{z}, \mathrm{t}) \text {, } \\
& \mathrm{M}(\mathrm{Tz}, \mathrm{z}, \mathrm{t})\} \\
& =\mathrm{M}(\mathrm{z}, \mathrm{Tz}, \mathrm{t}) \\
& \mathrm{n}\left(\mathrm{x}_{2 \mathrm{n}}, \mathrm{Tz}, \mathrm{t}\right)=\max \left\{\mathrm{N}(\mathrm{STTz}, \mathrm{QTz}, \mathrm{t}), \mathrm{N}\left(\mathrm{ABx}_{2 \mathrm{n}}, \mathrm{Px}_{2 \mathrm{n}}, \mathrm{t}\right),\right. \\
& \mathrm{N}\left(\mathrm{ABx}_{2 \mathrm{n}}, \mathrm{QTz}, \alpha \mathrm{t}\right), \mathrm{N}\left(\mathrm{STTz}, \mathrm{Px}_{2 \mathrm{n}}, \mathrm{t}\right), \\
& \left.\left.\mathrm{N}\left(\mathrm{STT}_{\mathrm{N}}, \mathrm{Px}_{2 \mathrm{n}}, \mathrm{t}\right)\right)\right\}
\end{aligned}
$$

Letting $n \rightarrow \infty$ we get,

$\mathrm{n}(\mathrm{z}, \mathrm{Tz}, \mathrm{t})=\max \{\mathrm{N}(\mathrm{Tz}, \mathrm{Tz}, \mathrm{t}), \mathrm{N}(\mathrm{ABz}, \mathrm{Pz}, \mathrm{t})$,

$$
\begin{aligned}
& \mathrm{N}(\mathrm{ABz}, \mathrm{Tz}, \mathrm{t}), \mathrm{N}(\mathrm{Tz}, \mathrm{Pz}, \mathrm{t}), \mathrm{N}(\mathrm{Tz}, \mathrm{ABz}, \mathrm{t})\} \\
= & \max \{0, \mathrm{~N}(\mathrm{z}, \mathrm{z}, \mathrm{t}), \mathrm{N}(\mathrm{z}, \mathrm{Tz}, \mathrm{t}), \mathrm{N}(\mathrm{Tz}, \mathrm{z}, \mathrm{t}), \mathrm{N}(\mathrm{Tz}, \mathrm{z}, \mathrm{t})\} \\
= & \max \{0,0, \mathrm{~N}(\mathrm{z}, \mathrm{Tz}, \mathrm{t}), \mathrm{N}(\mathrm{Tz}, \mathrm{z}, \mathrm{t}), \mathrm{N}(\mathrm{Tz}, \mathrm{z}, \mathrm{t})\} \\
= & \mathrm{N}(\mathrm{z}, \mathrm{Tz}, \mathrm{t})
\end{aligned}
$$

By using Lemma 1.14, we get $\mathrm{Tz}=\mathrm{z}$

Now $\mathrm{z}=\mathrm{Pz}=\mathrm{ABz}=\mathrm{Qz}=\mathrm{STz}=\mathrm{Tz}$.

$$
\begin{aligned}
& \Rightarrow \mathrm{STz}=\mathrm{Tz}=\mathrm{z} \\
& \Rightarrow \mathrm{Sz}=\mathrm{z}
\end{aligned}
$$

Hence, $\quad \mathrm{Sz}=\mathrm{Tz}=\mathrm{Qz}=\mathrm{z}$.

We now claim that $\mathrm{Bz}=\mathrm{z}$, for this we take $\mathrm{x}=\mathrm{Bz}, \mathrm{y}=\mathrm{x}_{2 \mathrm{n}+1}$ and using (ii), we get

$$
\left.\int_{0}^{M(P B z, Q x}{ }^{2 n+1}, k t\right) d t \geq \int_{0}^{m\left(B z, x_{2 n+1}, t\right)} \varphi(t) d t,
$$

$N\left(P B z, Q x_{2 n+1}, k t\right)$

$$
\int_{0}^{2 n+1} \varphi(t) d t \leq \int_{0}^{n\left(B z, x_{2 n+1}, t\right)} \varphi(t) d t
$$

$N\left(P B z, Q x_{2 n+1}, k t\right) \quad n\left(B z, x_{2 n+1}, t\right) \quad$ Take $\alpha=1$

$$
\int_{0}^{2 n+1} \varphi(t) d t \leq \int_{0}^{n\left(B z, x_{2 n+1}, t\right)} \varphi(t) d t
$$

$\mathrm{m}\left(\mathrm{Bz}, \mathrm{x}_{2 \mathrm{n}+1}, \mathrm{t}\right)=\min \left\{\mathrm{M}\left(\mathrm{STx}_{2 \mathrm{n}+1}, \mathrm{Qx}_{2 \mathrm{n}+1}, \mathrm{t}\right), \mathrm{M}(\mathrm{ABBz}, \mathrm{PBz}, \mathrm{t})\right.$,

$$
\mathrm{M}\left(\mathrm{ABBz}, \mathrm{Qx}_{2 \mathrm{n}+1}, \mathrm{t}\right), \mathrm{M}\left(\mathrm{STx}_{2 \mathrm{n}+1}, \mathrm{PBz}, \mathrm{t}\right),
$$$$
\left.\mathrm{M}\left(\mathrm{STx}_{2 \mathrm{n}+1}, \mathrm{ABBz}, \mathrm{t}\right)\right\}
$$

As $\mathrm{BP}=\mathrm{PB}$ and $\mathrm{AB}=\mathrm{BA}$ we have $\mathrm{PBz}=\mathrm{BPz}=\mathrm{Bz}$ and $\mathrm{AB}(\mathrm{Bz})$ $=\mathrm{B}(\mathrm{AB}) \mathrm{z}=\mathrm{Bz}$.

Letting $n \rightarrow \infty$ we get,

$\mathrm{m}(\mathrm{Bz}, \mathrm{z}, \mathrm{t})=\min \{\mathrm{M}(\mathrm{STz}, \mathrm{Qz}, \mathrm{t}), \mathrm{M}(\mathrm{ABBz}, \mathrm{PBz}, \mathrm{t})$,

$$
\begin{aligned}
& \mathrm{M}(\mathrm{ABBz}, \mathrm{Qz}, \mathrm{t}, \mathrm{M}(\mathrm{STz}, \mathrm{PBz}, \mathrm{t}), \\
& \mathrm{M}(\mathrm{STz}, \mathrm{ABBz}, \mathrm{t})\} \\
= & \min \{\mathrm{M}(\mathrm{z}, \mathrm{z}, \mathrm{t}), \mathrm{M}(\mathrm{Bz}, \mathrm{Bz}, \mathrm{t}), \mathrm{M}(\mathrm{Bz}, \mathrm{z}, \mathrm{t}), \\
& \mathrm{M}(\mathrm{z}, \mathrm{Bz}, \mathrm{t}), \mathrm{M}(\mathrm{z}, \mathrm{Bz}, \mathrm{t})\} \\
= & \min \{1,1, \mathrm{M}(\mathrm{Bz}, \mathrm{z}, \mathrm{t}), \mathrm{M}(\mathrm{z}, \mathrm{Bz}, \mathrm{t}), \mathrm{M}(\mathrm{z}, \mathrm{Bz}, \mathrm{t})\} \\
= & \mathrm{M}(\mathrm{B} z, \mathrm{z}, \mathrm{t})
\end{aligned}
$$

$\mathrm{n}\left(\mathrm{Bz}, \mathrm{x}_{2 \mathrm{n}+1}, \mathrm{t}\right)=\max \left\{\mathrm{N}\left(\mathrm{STx}_{2 \mathrm{n}+1}, \mathrm{Qx}_{2 \mathrm{n}+1}, \mathrm{t}\right), \mathrm{N}(\mathrm{ABBz}, \mathrm{PBz}, \mathrm{t})\right.$,

$$
\begin{aligned}
& \mathrm{N}\left(\mathrm{ABBz}, \mathrm{Qx}_{2 \mathrm{n}+1}, \mathrm{t}\right), \mathrm{N}\left(\mathrm{STx}_{2 \mathrm{n}+1}, \mathrm{PBz}, \mathrm{t}\right), \\
& \left.\mathrm{N}\left(\mathrm{STx}_{2 \mathrm{n}+1}, \mathrm{ABBz}, \mathrm{t}\right)\right\}
\end{aligned}
$$

Letting $n \rightarrow \infty$ we get,

$$
\begin{aligned}
\mathrm{n}(\mathrm{Bz}, \mathrm{z}, \mathrm{t})= & \max \{\mathrm{N}(\mathrm{STz}, \mathrm{Qz}, \mathrm{t}), \mathrm{N}(\mathrm{ABBz}, \mathrm{PBz}, \mathrm{t}), \mathrm{N}(\mathrm{ABBz}, \mathrm{Qz}, \mathrm{t}), \\
& \mathrm{N}(\mathrm{STz}, \mathrm{PBz}, \mathrm{t}), \mathrm{N}(\mathrm{STz}, \mathrm{ABBz}, \mathrm{t})\} \\
= & \max \{\mathrm{N}(\mathrm{z}, \mathrm{z}, \mathrm{t}), \mathrm{N}(\mathrm{Bz}, \mathrm{Bz}, \mathrm{t}), \mathrm{N}(\mathrm{Bz}, \mathrm{z}, \mathrm{t}, \mathrm{N}(\mathrm{z}, \mathrm{Bz}, \mathrm{t}), \\
& \mathrm{N}(\mathrm{z}, \mathrm{Bz}, \mathrm{t})\} \\
= & \max \{0,0, \mathrm{~N}(\mathrm{Bz}, \mathrm{z}, \mathrm{t}), \mathrm{N}(\mathrm{z}, \mathrm{Bz}, \mathrm{t}), \mathrm{N}(\mathrm{z}, \mathrm{Bz}, \mathrm{t})\} \\
= & \mathrm{N}(\mathrm{Bz}, \mathrm{z}, \mathrm{t})
\end{aligned}
$$

By using Lemma1.14,we get

$$
\mathrm{Bz}=\mathrm{z}
$$

and $\mathrm{ABz}=\mathrm{z}$ implies $\mathrm{Az}=\mathrm{z}$. Therefore,

$$
\mathrm{Az}=\mathrm{Bz}=\mathrm{Pz}=\mathrm{z}
$$

From (2.16) and (2.18), we have

$$
\mathrm{Az}=\mathrm{Bz}=\mathrm{Pz}=\mathrm{Sz}=\mathrm{Tz}=\mathrm{Qz}=\mathrm{z} .
$$

It follows that $\mathrm{z}$ is a common fixed point of $\mathrm{P}, \mathrm{Q}, \mathrm{A}, \mathrm{B}, \mathrm{S}$ and $\mathrm{T}$.

Assume (iii) (b) 
Then from reciprocal continuity and compatibity of the pair $(\mathrm{Q}$, ST) and using(2.1), follows that

$$
\mathrm{Bz}=\mathrm{Tz} \text {. }
$$

Since $\mathrm{Q}(\mathrm{X}) \subseteq \mathrm{AB}(\mathrm{X})$ there exist $\mathrm{u} \in \mathrm{X}$ such that $\mathrm{Qz}=\mathrm{ABw}$. We now claim that $\mathrm{Pw}=\mathrm{ABw}$.

using(ii), we get

$$
\begin{gathered}
\int_{0}^{M(P w, A B w, k t)} \varphi(t) d t \geq \int_{0}^{m(w, w, t)} \varphi(t) d t \\
\int_{0}^{M(P w, A B w, k t)} \varphi(t) d t=\int_{0}^{M(A w, Q z, k t)} \varphi(t) d t \geq \int_{0}^{m(w, z, t)} \varphi(t) d t
\end{gathered}
$$$$
\int_{0}^{N(A w, S w, k t)} \varphi(t) d t=\int_{0}^{N(A w, B z, k t)} \varphi(t) d t \leq \int_{0}^{n(w, z, t)} \varphi(t) d t
$$$$
\int_{0}^{N(P w, A B w, k t)} \varphi(t) d t \leq \int_{0}^{n(w, w, t)} \varphi(t) d t
$$$$
\int_{0}^{N(P w, A B w, k t)} \varphi(t) d t=\int_{0}^{N(P w, Q z, k t)} \varphi(t) d t \leq \int_{0}^{n(w, z, t)} \varphi(t) d t
$$

Take $\alpha=1$

$$
\begin{aligned}
\mathrm{m}(\mathrm{w}, \mathrm{z}, \mathrm{t})=\min \{\mathrm{M}(\mathrm{STz}, \mathrm{Qz}, \mathrm{t}), \mathrm{M}(\mathrm{ABw}, \mathrm{Pw}, \mathrm{t}), \\
\mathrm{M}(\mathrm{ABw}, \mathrm{Qz}, \mathrm{t}), \mathrm{M}(\mathrm{STz}, \mathrm{Pw}, \mathrm{t}) \\
\mathrm{M}(\mathrm{STz}, \mathrm{ABw}, \mathrm{t})\} \\
=\min \{\mathrm{M}(\mathrm{ABw}, \mathrm{ABw}, \mathrm{t}), \mathrm{M}(\mathrm{ABw}, \mathrm{Pw}, \mathrm{t}), \\
\mathrm{M}(\mathrm{Qz}, \mathrm{Qz}, \mathrm{t}), \mathrm{M}(\mathrm{ABw}, \mathrm{Pw}, \mathrm{t}), \\
\mathrm{M}(\mathrm{ABw}, \mathrm{ABw}, \mathrm{t})\} \\
=\min \{1, \mathrm{M}(\mathrm{ABw}, \mathrm{Pw}, \mathrm{t}), 1, \mathrm{M}(\mathrm{ABw}, \mathrm{Pw}, \mathrm{t}), 1\} \\
=\mathrm{M}(\mathrm{Pw}, \mathrm{ABw}, \mathrm{t})
\end{aligned}
$$

$\mathrm{n}(\mathrm{w}, \mathrm{z}, \mathrm{t})=\max \{\mathrm{N}(\mathrm{STz}, \mathrm{Qz}, \mathrm{t}), \mathrm{N}(\mathrm{ABw}, \mathrm{Pw}, \mathrm{t}), \mathrm{N}(\mathrm{ABw}, \mathrm{Qz}, \mathrm{t})$,

$$
\begin{aligned}
&\mathrm{N}(\mathrm{STz}, \mathrm{Pw}, \mathrm{t}) \mathrm{N}(\mathrm{STz}, \mathrm{ABw}, \mathrm{t})\} \\
&= \max \{\mathrm{N}(\mathrm{ABw}, \mathrm{ABw}, \mathrm{t}), \mathrm{N}(\mathrm{ABw}, \mathrm{Pw}, \mathrm{t}), \\
& \mathrm{N}(\mathrm{Qz}, \mathrm{Qz}, \mathrm{t}), \mathrm{N}(\mathrm{ABw}, \mathrm{Pw}, \mathrm{t}), \\
& \mathrm{N}(\mathrm{ABw}, \mathrm{ABw}, \mathrm{t}) \\
&=\max \{0, \mathrm{~N}(\mathrm{ABw}, \mathrm{Pw}, \mathrm{t}), 0, \mathrm{~N}(\mathrm{ABw}, \mathrm{Pw}, \mathrm{t}), 0\} \\
&=\mathrm{N}(\mathrm{Pw}, \mathrm{ABw}, \mathrm{t})
\end{aligned}
$$

By using Lemma 1.14, we get $\mathrm{Pw}=\mathrm{ABw}$

Thus $\mathrm{Pw}=\mathrm{ABw}=\mathrm{Qz}=\mathrm{STz}$.

Now the rest of the proof runs on the same lines from(2.8).

Let $\mathrm{u}$ and $\mathrm{z}$ be two distinct common fixed points of $\mathrm{P}, \mathrm{Q}, \mathrm{A}, \mathrm{B}, \mathrm{S}$ and $\mathrm{T}$ then from (2.1),we have

$$
\int_{0}^{M(P u, Q z, k t)} \phi(t) d t \geq \int_{0}^{m(u, z, t)} \phi(t) d t
$$

where

$$
\begin{aligned}
& \mathrm{m}(\mathrm{u}, \mathrm{z}, \mathrm{t})= \min \{\mathrm{M}(\mathrm{STz}, \mathrm{Qz}, \mathrm{t}), \mathrm{M}(\mathrm{ABu}, \mathrm{Pu}, \mathrm{t}), \\
& \mathrm{M}(\mathrm{ABu}, \mathrm{Qz}, \alpha \mathrm{t}), \mathrm{M}(\mathrm{STz}, \mathrm{Pu},(2-\alpha) \mathrm{t}), \\
&\mathrm{M}(\mathrm{STz}, \mathrm{ABu}, \mathrm{t})\} \\
& \mathrm{m}(\mathrm{u}, \mathrm{z}, \mathrm{t})=\min \{1,1, \mathrm{M}(\mathrm{Pu}, \mathrm{Qz}, \alpha \mathrm{t}), \mathrm{M}(\mathrm{Qz}, \mathrm{Pu},(2-\alpha) \mathrm{t}), \\
&\mathrm{M}(\mathrm{Qz}, \mathrm{ABu}, \mathrm{t})\} \\
& \mathrm{m}(\mathrm{u}, \mathrm{z}, \mathrm{t})=\min \{1,1, \mathrm{M}(\mathrm{Pu}, \mathrm{Qz}, \alpha \mathrm{t}), \mathrm{M}(\mathrm{Qz}, \mathrm{Pu},(2-\alpha) \mathrm{t}), \\
&\mathrm{M}(\mathrm{Qz}, \mathrm{Pu}, \mathrm{t})\}
\end{aligned}
$$

$$
\int_{0}^{M(P u, Q z, k t)} \varphi(t) d t \geq \int_{0}^{M(P u, Q z, t)} \varphi(t) d t .
$$

Now from (2.2),we have

$$
\int_{0}^{N(P u, Q z, k t)} \varphi(t) d t \leq \int_{0}^{n(u, z, t)} \varphi(t) d t
$$

where

$$
\begin{aligned}
& \mathrm{n}(\mathrm{u}, \mathrm{z}, \mathrm{t})=\max \{\mathrm{N}(\mathrm{STz}, \mathrm{Qz}, \mathrm{t}), \mathrm{N}(\mathrm{ABu}, \mathrm{Pu}, \mathrm{t}), \mathrm{N}(\mathrm{ABu}, \mathrm{Qz}, \alpha \mathrm{t}), \\
& \mathrm{N}(\mathrm{STz}, \mathrm{Pu},(2-\alpha) \mathrm{t}), \mathrm{N}(\mathrm{STz}, \mathrm{ABu}, \mathrm{t})\} \\
& \mathrm{n}(\mathrm{u}, \mathrm{z}, \mathrm{t})=\max \{1,1, \mathrm{~N}(\mathrm{Pu}, \mathrm{Qz}, \alpha \mathrm{t}), \mathrm{N}(\mathrm{Qz}, \mathrm{Pu},(2-\alpha) \mathrm{t}), \\
& \mathrm{N}(\mathrm{Qz}, \mathrm{ABu}, \mathrm{t})\} \\
& \mathrm{n}(\mathrm{u}, \mathrm{z}, \mathrm{t})=\max \{1,1, \mathrm{~N}(\mathrm{Pu}, \mathrm{Qz}, \alpha \mathrm{t}), \mathrm{N}(\mathrm{Qz}, \mathrm{Pu},(2-\alpha) \mathrm{t}), \\
& \mathrm{N}(\mathrm{Qz}, \mathrm{Pu}, \mathrm{t})\} \\
& \int_{0}^{N(P u, Q z, k t)} \varphi(t) d t \leq \int_{0}^{N(P u, Q z, t)} \varphi(t) d t
\end{aligned}
$$

By using lemma 1.14, we have

$$
\begin{aligned}
\mathrm{Pu} & =\mathrm{Qz} \\
\Rightarrow \mathrm{u} & =\mathrm{z} .
\end{aligned}
$$

This completes the proof of the theorem.

\section{ACKNOWLEDGMENTS}

The author is very thankful to reviewers for their valuable suggestions

\section{REFERENCES}

[1] A. Branciari, A fixed point theorem for mappings satisfying a general condition of integral type, International Journal of Mathematics and mathematical science,29(2002),531-536.

[2] B. Schweizer and A. Sklar, statistical spaces, Pacific Journal of Mathematics, 10(1960),313-334.

[3] C.Alaca, D.Turkoglu and C. Yildiz, Fixed points in intuitionistic fuzzy metric spaces, Choas. Solitons and Fractals, 29(2006),1073-1078. 
[4] K. Atanassov, intuitionistic fuzzy sets, Fuzzy sets and systems 20(1986), 87-96.

[5] L.A. Zadeh , Fuzzy sets, inform and control 8(1965),338353.

[6] O. Kramosial and J. Michalek, Fuzzy metric and statistical metric spaces, Kybernetica, 11(1975),330-334.

[7] S.Muralisankar and G. Kalpana, Common Fixed Point Theorem in Intuitionistic Fuzzy Metric Space using
General Contractive Condition of Integral Type, Int. J. Contemp. Math. Sciences, 4(2009), 505-518.

[8] S. Sharma, S. Kutukcu and R.S.Rathore, Common fixed point for multivalued mappings in intuitionistic fuzzy metric space, Communication of Korean Mathematical Society, 22(3)(2007), 391-399.

[9] E.P. Klement, R.Mesiar, E. Pap, Triangular norms ,Kluwer Acad. Pupl., Dordrecht,2000. 